\title{
Ultrafast Formation of Nonemissive Species via Intermolecular Interaction in Single Crystals of Conjugated Molecules
}

\author{
M. A. Loi, A. Mura, and G. Bongiovanni \\ Dipartimento di Fisica, Università di Cagliari, and Istituto Nazionale per la Fisica della Materia, I-09042 Monserrato, Italy \\ Q. Cai, C. Martin, H. R. Chandrasekhar, and M. Chandrasekhar \\ Department of Physics and Astronomy, University of Missouri, Columbia, Missouri 65211 \\ W. Graupner* \\ Department of Physics, Virginia Tech, Blacksburg, Virginia 24061-0435 \\ F. Garnier \\ Laboratoires des Materiaux Moléculaires (CNRS), Thiais, France
}

(Received 19 May 2000)

\begin{abstract}
We investigate the influence of interchain interactions on the photoluminescence processes in a sexithiophene single crystal by applying hydrostatic pressure. We perform transient photoluminescence spectroscopy in the time domain of $100 \mathrm{fs}$ for pressures up to $60 \mathrm{kbar}$. The combined use of steady-state and time-resolved optical spectroscopies allows us to show that the pressure-induced quenching of the photoluminescence is caused by an ultrafast ( $\sim 100 \mathrm{fs})$ formation of intermolecular species.
\end{abstract}

DOI: 10.1103/PhysRevLett.86.732

$\pi$-conjugated polymers and oligomers are an emerging class of new semiconductors to realize low cost electronic and optoelectronic devices, such as light emitting diodes, lasers, field effect transistors, and photovoltaic cells [1]. High-quality single crystals of oligothiophenes and polyacenes exhibit the highest carrier mobilities among organic semiconductors, up to a few $\mathrm{cm}^{2} \mathrm{~V}^{-1} \mathrm{~s}^{-1}$ at room temperature [2] and up to $10^{3}-10^{5} \mathrm{~cm}^{2} \mathrm{~V}^{-1} \mathrm{~s}^{-1}$ at low temperatures [3]. These exceptionally good transport properties have permitted the demonstration of the first electrically pumped organic laser [4].

The device development has been systematically accompanied and boosted by a basic research aimed at clarifying the photophysics of these materials [5]. A central issue concerns the influence of interchain interactions on the photoluminescence (PL) efficiency. In the simplest case, PL can be viewed as the temporal sequence of three elementary processes: generation of the primary electronhole pairs, energy relaxation towards one or more excited species, and finally radiative electron-hole recombination. The PL efficiency critically depends on the branching ratios among the different photoexcited species and their nature. In $\pi$-conjugated oligomer crystals, the lowest energy excitations are excitons with the electronhole pair confined in the same chain and binding energies of typically several tenths of eV [6]. Excitons with the electron-hole wave function delocalized over more chains are known as charge-transfer states [7]. Because of the larger electron-hole separation, these states can be considered as a first step towards uncorrelated polaron generation. The formation of such nonluminescent species is detrimental for the PL quantum yield [8]. Evidences for both ultrafast hot charge separation [9] and free electron-
PACS numbers: 78.47.+p, 78.55.Kz, 78.66.Qn

hole pair generation from thermalized excitons have been reported [10]. The nature of the process is, however, controversial, since the formation of intermolecular excitations seems to be favored by disorder [7], or chemical impurities [11].

The intermolecular coupling can be directly controlled by varying the interchain distances through applied pressure. Most of the previous studies performed under pressure employs steady-state techniques, which provide only limited information on the dynamics of those processes which determine PL efficiency, i.e., energy relaxation, exciton diffusion, and decay. Pioneering work on the photoexcitation dynamics under high pressure was done by Hess et al. in conjugated polymers [12] and in molecular solids [13], by using time-resolved pump-probe techniques. No intrinsic effect of pressure on the photoexcitation relaxation was observed, the changes found in the transient decays being ascribed to a pressure-induced variation of the density of nonradiative defects [12], or to disorder effects [13].

In this Letter, we study the influence of interchain interaction on the PL process in a sexithiophene single crystal [14]. The high structural order [15] and purity [2] of the single crystal permits the investigation of the intrinsic effects due to interchain interaction. By applying pressure we regulate the strength of the intermolecular coupling. The buildup and decay of the low energy state population is directly probed by 100 -femtosecond PL spectroscopy. This way, we acquire a more direct access to the exciton dynamics with respect to pump-probe experiments [13], in which the interplay between excited state absorption/ emission and bleaching can give rise to controversial interpretations. The variation with pressure of the light 
intensity emitted in the first few hundreds of femtoseconds provides a quantitative evaluation of the effect of interchain interaction on the generation yield of excitons. The present experiments provide new information about the intrinsic mechanisms of formation of interchain excitations [16] and, in particular, when they are formed and how their generation rate depends on the interchain interaction strength.

Sexithiophene (T6) single crystals were grown by sublimation under argon atmosphere [14]. Pressure studies were conducted in a diamond anvil cell with cryogenically loaded argon or ethanol-methanol mixture as pressure medium and ruby PL as pressure calibrant [17]. We have employed the nonlinear up-conversion technique to temporally resolve the PL signal [18]. The output of a mode locked Ti:sapphire tuned at $1.53 \mathrm{eV}$ is split into two parts. One part is frequency doubled to excite the sample. The other part (the gate pulse) is sent through a variable delay line and then mixed with the sample PL in a $0.5 \mathrm{~mm}$-thick- $\beta$-barium borate crystal to generate the up-conversion signal. The full width at half maximum (FWHM) of the autocorrelation trace of the gate pulse is 160 fs. The cross correlation FWHM between the gate pulse and its second harmonic is $200 \mathrm{fs}$, and increases up to $220 \mathrm{fs}$ passing through the diamonds. The excitation power was kept below $3 \mathrm{~mW}$ over a spot diameter of $50 \mu \mathrm{m}$. At ambient pressure, the sample was held under vacuum. Under this condition and in the diamond cell, no degradation of the sample was detected. It is important to point out that all the changes of the PL induced by pressure were found to be reversible.

In Fig. 1(a), we present the evolution of the PL spectra of the T6 single crystal as a function of temperature. The spectrum at $15 \mathrm{~K}$ shows a fine structure on top of a broad background. The broad structures are due to light emission from aggregates, i.e., traps due to molecules with different packing with respect to the bulk [7], while the sharp features result from radiative decay of very shallow trapped excitons [15]. The faint $0-0$ emission of the latter is peaked at $2.271 \mathrm{eV}, 5 \mathrm{meV}$ below the transition energy to the lowest free exciton band [15]. This band becomes thermally populated with increasing temperature. At $300 \mathrm{~K}$, thermal detrapping of excitons gives rise to a pure free exciton recombination. Although the lowest lying exciton band has ungerade symmetry and thus is dipole allowed, its oscillator strength is very weak. The unusual intensity distribution among the 0-0 emission and its vibronic replicas has been described in terms of the Herzberg-Teller mechanism [7]. The possibility of observing the free exciton emission at room temperature results from the very low percentage of structural defects present in the single crystal [2]. Conversely, the density of defects in thin films is such that aggregate emission dominates even at room temperature [7].

To determine the time $\tau$ taken by the hot photoexcitations to relax down to the lowest lying free exciton band, we have monitored the population of the latter by study-

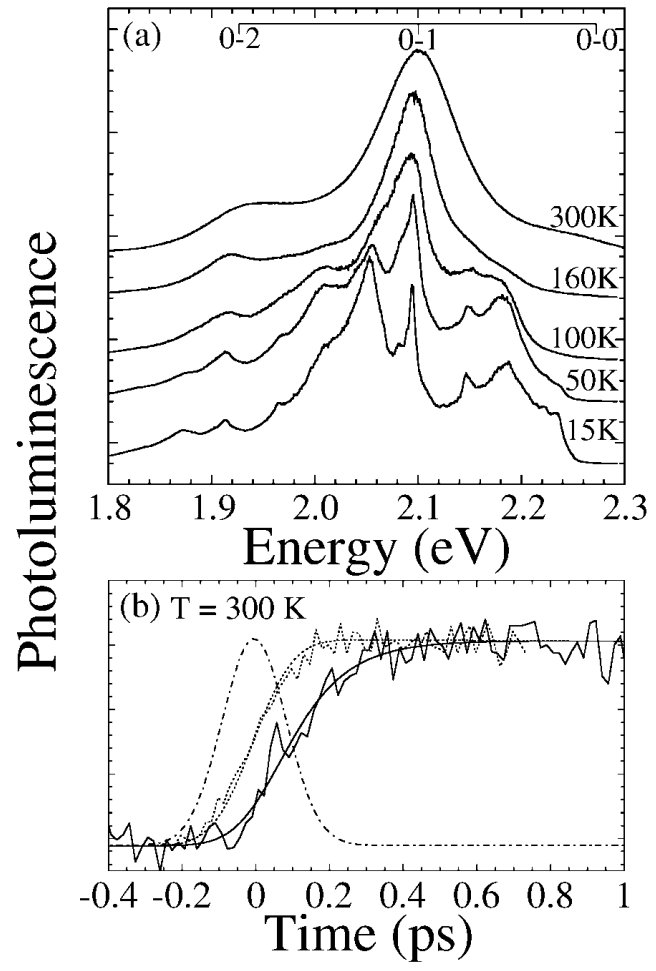

FIG. 1. (a) Evolution of the PL spectra of the T6 single crystal with temperature. (b) The scattered lines are the time resolved PL signal of a T6 single crystal (solid line) and a coumarin dye (dashed line). The dot-dashed line is the cross correlation signal between the pump pulse going through the sample and the gate pulse. The smooth solid line is a least-squares fit to the up-conversion signal of T6. The smooth dashed line represents the shortest simulated rise time of our experimental apparatus.

ing the buildup of the vibronic emission at $2.1 \mathrm{eV}$. The transients have been measured by using the optical gate technique and are shown in Fig. 1(b). To provide a visual evaluation of the fastest rise time that can be detected by our experimental setup, the ultrafast buildup of the PL of a coumarin dye (emission energy of $2.45 \mathrm{eV}$ ) is also reported in Fig. 1(b). The transient is well reproduced by the simulated up-conversion signal of a steplike emission $(\tau=0)$ [19]. The growth of the T6 PL is very fast, but slower than the reference dye signal. A quantitative estimate of the emission rise time, $\tau=120 \pm 20 \mathrm{fs}$, has been obtained by performing a least-squares fit to the experimental data which accounts for the finite time resolution of the experimental apparatus [19]. During the time $\tau$, the excess photon energy $\Delta E_{\text {ex }}(790 \mathrm{meV})$ is released to the thermal bath with a rate $\langle P\rangle=\Delta E_{\mathrm{ex}} / \tau=6.6 \pm 1.3 \mathrm{meV} /$ fs. We compare $\langle P\rangle$ with the estimate of the maximum energy relaxation rate $P_{M} \sim h \nu^{2}$ between vibronic levels [20]. For the C-C mode of T6, most strongly coupled to the electronic excitations, $h \nu=156 \mathrm{meV}, P_{M} \sim 5.9 \mathrm{meV} / \mathrm{fs}$, in excellent agreement with the experimental value $\langle P\rangle$.

Figure 2 shows the T6 PL spectra for different pressures. The spectra show a rigid linear redshift (inset of Fig. 3) on going from ambient pressure to $55 \mathrm{kbar}$ with a rate of 


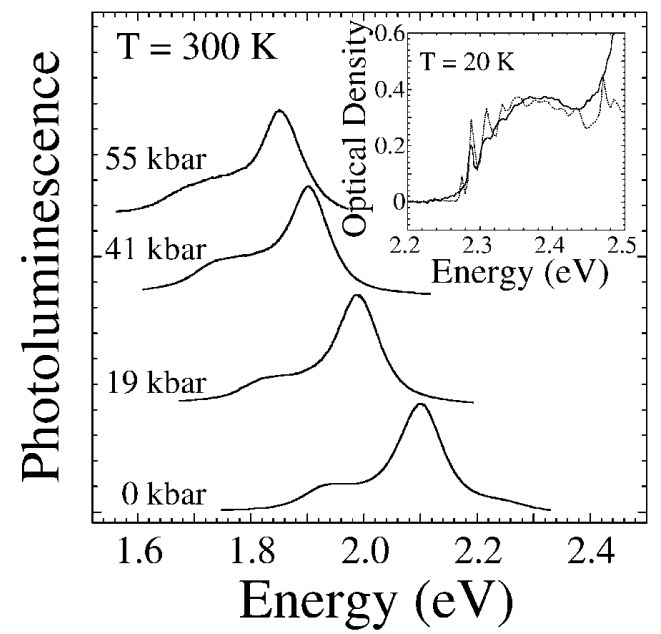

FIG. 2. PL spectra of T6 single crystal at different pressures. All spectra are normalized to the maximum and are displaced vertically for clarity. Inset: Optical density of the T6 single crystal at ambient pressure (dashed line) and $20 \mathrm{kbar}$ (solid line). The $20 \mathrm{kbar}$ spectrum is blueshifted to be at the same energy of the ambient pressure one.

$-4.1 \mathrm{meV} / \mathrm{kbar}$. The decrease of the optical gap is comparable with that found in similar materials [21]. Besides a slight broadening, the shape of the spontaneous emission does not change, indicating that free exciton emission persists and dominates even at the highest pressures.

The behavior of the cw PL intensity as a function of pressure and normalized to the absorbed photons is reported in Fig. 3; data are shown for the 0-1 emission and for the spectrally integrated signal. From ambient pressure to $60 \mathrm{kbar}$, the intensity of the overall spectrum reduces by roughly a factor of 10 . Different physical processes have been suggested to explain the quenching of the PL under

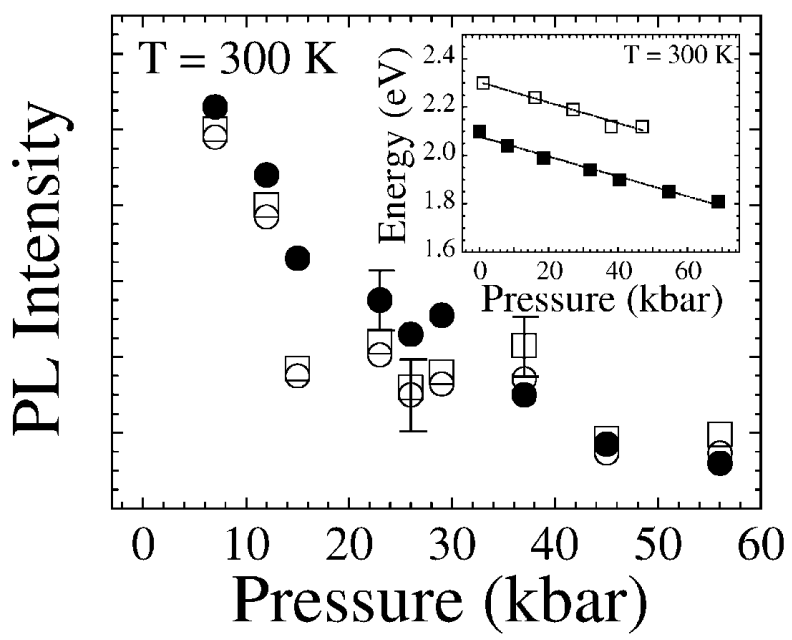

FIG. 3. cw intensity (empty squares) of the 0-1 peak emission and of the spectrally integrated PL signal (empty circles). Full circles: intensity of the $0-1$ peak emission at 1 ps delay. All the values are scaled to coincide at ambient pressure. Inset: energy of the 0-1 emission (full squares) and of the absorption onset (empty squares) versus pressure. high pressure [21]. The mechanisms proposed are due to extrinsic properties related to the presence of nonradiative centers or to intrinsic properties of the material, like the formation of excited states having a weak or nonradiative coupling to the ground state, namely, charge-transfer excitons and excimers [22].

Charge-transfer excitons and uncorrelated polaron pairs can be generated efficiently from singlet excitons during thermalization [9]. In order to distinguish between hot excited state branching and thermalized exciton decay channels, we have recorded the PL transients as a function of pressure. Figure 3 shows that the intensity of the $0-1$ emission taken at $t=1 \mathrm{ps}$ - just after the initial fast relaxation time $\tau$ but before any slow decay takes place - undergoes the same reduction with pressure found in the timeintegrated experiments. Moreover, the decay of the up-conversion signal of the 0-1 emission shown in Fig. 4 is clearly unaffected by the decrease of the interchain distance [13]. The PL decay of the spectrally integrated PL, recorded on a much longer temporal scale through a streak camera and shown in the inset of Fig. 4, also exhibits a very weak dependence on pressure [23]. These results imply that the PL quenching can be due to either a reduction of the radiative emission rate or a decrease of the population of thermalized excitons caused by a hot excited state branching.

The lower PL efficiency could be symptomatic of the formation of excimers, i.e., dimers that exist only in the excited state [22]. They provoke substantial changes in the PL spectra and in the PL long time decay rates. All the previous experimental findings are against this scenario. Moreover, the energy shift with pressure of excimers should be different from that of free excitons [24]. In T6,

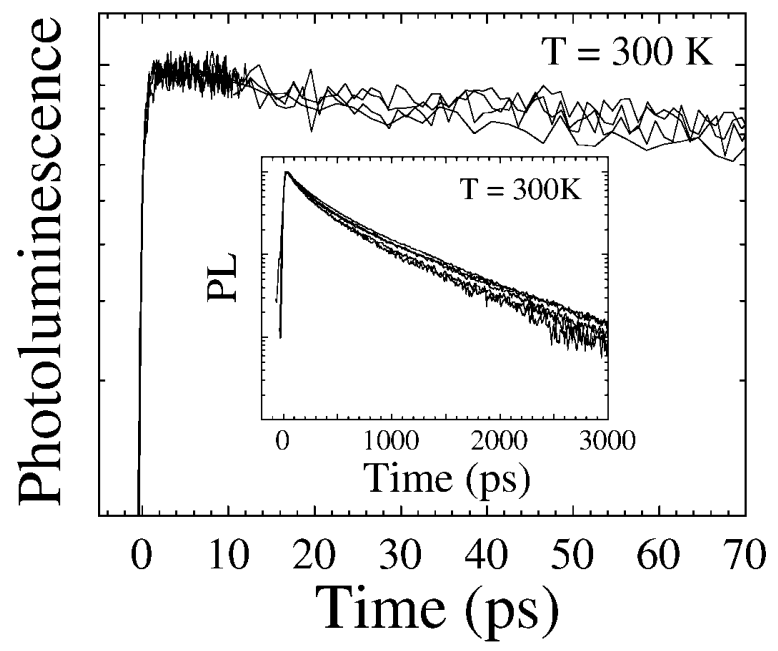

FIG. 4. Time-resolved decays of the 0-1 emission taken with the up-conversion technique, at ambient pressure, 7, 25, and 37 kbar. Inset: Time-resolved decays of the spectrally integrated PL emission, taken on a longer scale through a streak camera, at ambient pressure, 7, 25, 37, and 56 kbar. All the decays are normalized to the maximum intensity. 
the redshift of the PL spectra shown in the inset of Fig. 3 and that of the lowest energy absorption peak are the same. We therefore conclude that excimers are not formed when pressure is applied.

The radiative decay rate is proportional to the optical transition matrix elements that connect the initial and final states in the light emission process. The same matrix elements govern photon absorption and emission. In the inset of Fig. 2, we report the absorption spectra at ambient pressure and $20 \mathrm{kbar}$ that show the onset transition to the lowest energy exciton. We observe broadening with pressure; however, the photon absorption cross section does not decrease under pressure. Consequently, we conclude that the strong decrease of the PL quantum yield is mostly due to an initial branching of the primary photoexcitons into both intramolecular excitons and nonemissive species. We suggest the latter to be interchain electron-hole pairs that are favored at shorter interchain distances.

At ambient pressure, the lowest unoccupied molecular orbital of symmetry $b_{u}$ gives rise in the crystal to four exciton bands of symmetry $a_{u}, a_{g}, b_{u}$, and $b_{g}$, respectively [7]. Intermolecular interactions between nonequivalent molecules within a single layer lift the degeneracy of the $a$ and $b$ states (the split states are called Davydov excitons [25]). The weak transition to the lower $a_{u}$ states is at $2.276 \mathrm{eV}$, while the one towards the upper exciton levels $b_{u}$ is at $2.592 \mathrm{eV}$. The weak interlayer coupling is responsible for the small energy difference between ungerade and gerade levels [7]. Charge-transfer states have been located at $2.78 \mathrm{eV}$ [2].

The shortening of the interchain distance reduces the Coulomb energy required to split the Frenkel excitons into intermolecular excitations; this leads to an efficient mixing between the charge-transfer states and the closely lying upper Davydov excitons [15]. An almost complete mixing between these two levels is confirmed by quantum mechanical calculations performed on T6 clusters with varying interchain distance [26]. Hence, we suggest that our experiments are indicative for (1) an enhanced generation of polarons due to the stronger intermolecular character of the primary excitations which facilitates electron-hole separation, and, on the other hand, (2) a reduced probability for the excitons to relax towards the lower Davydov levels, from which light emission occurs.

In conclusion, we have investigated the response of a sexithiopene single crystal to pressure by applying femtosecond photoluminescence and cw-optical spectroscopies. Upon increasing interchain interaction, we have found a considerable reduction of the photoluminescence efficiency, but a negligible change of the exciton lifetime. We show that the photoluminescence quenching is due to a decay of the hot primary photoexcitations into nonemissive interchain excitations, and not to splitting of thermalized excitons. At the highest pressures, the formation of interchain species is the dominant relaxation channel.

We thank J. Cornil and J.Ph. Calbert for discussions. The work was partially supported by the University of Missouri Research Board and the OeNB Project No. 6608.

*Present address: eMagin Corporation, 2070 Route 52, Hopewell Junction, NY 12533.

[1] R. H. Friend et al., Nature (London) 397, 121 (1999); A. Dodabalapur et al., Science 268, 270 (1995); J. H. Schön et al., Nature (London) 403, 408 (2000).

[2] J.H. Schön et al., Appl. Phys. Lett. 73, 3574 (1998); G. Horowitz et al., Phys. Rev. B 59, 10651 (1999).

[3] J. H. Schön et al., Science 288, 2338 (2000).

[4] J.H. Schön et al., Science 289, 599 (2000).

[5] Primary Photoexcitations in Conjugated Polymers: Molecular Exciton versus Band Model, edited by N.S. Sariciftci (World Scientific, Singapore, 1997).

[6] M. Knupfer et al., Phys. Rev. Lett. 83, 1443 (1999).

[7] C. Taliani et al., in Semiconducting Polymers: Chemistry, Physics, and Engineering, edited by G. Hadziioannou and P. F. van Hutten (Wiley \& Sons, New York, 2000).

[8] M. Yan et al., Phys. Rev. Lett. 75, 1992 (1995).

[9] W. Graupner et al., Phys. Rev. Lett. 81, 3259 (1998); M. Wohlgenannt et al., Phys. Rev. Lett. 82, 3344 (1999); V. I. Arkhipov et al., Phys. Rev. Lett. 82, 1321 (1999).

[10] G. Lanzani et al., Phys. Rev. Lett. 79, 3066 (1997).

[11] M. Yan et al., Phys. Rev. Lett. 73, 744 (1994); M. A. Loi et al., Phys. Rev. B 61, 1859 (2000).

[12] B. C. Hess et al., Phys. Rev. Lett. 66, 2364 (1991).

[13] B. C. Hess et al., Phys. Rev. B 50, 4871 (1994).

[14] G. Horowitz et al., Chem. Mater. 7, 1337 (1995).

[15] M. Muccini et al., J. Chem. Phys. 109, 10513 (1998).

[16] J. Cornil et al., J. Am. Chem. Soc. 120, 1289 (1998).

[17] A. Jayaraman, Rev. Sci. Instrum. 57, 1013 (1986).

[18] R. Kersting et al., Phys. Rev. Lett. 70, 3820 (1993); G. R. Hayes et al., Phys. Rev. B 52, R11 569 (1995).

[19] The temporal response of the experimental apparatus has been accounted for by making a convolution between the function $1-\exp (-t / \tau)$ and the up-conversion signal of the exciting second harmonic pulse.

[20] Z. Vardeny and J. Tauc, in Semiconductors Probed by Ultrafast Laser Spectroscopy, edited by R. R. Alfano (Academic Press Inc., New York, 1984), Vol. II.

[21] S. Webster et al., Polymer 37, 4961 (1996).

[22] M. Pope and C. E. Swenberg, Electronic Processes in Organic Crystals (Oxford University Press, New York, 1982).

[23] The slight increase of the decay rate gives a reduction of the time-integrated PL intensity of $\sim 20 \%$.

[24] M. Kobayashi et al., J. Phys. Soc. Jpn. 58, 809 (1989).

[25] A.S. Davydov, Theory of Molecular Excitons (Plenum Press, New York, 1971).

[26] J. Cornil et al. (unpublished). 